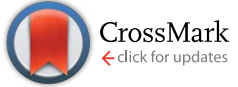

Cite this: Chem. Sci., 2015, 6, 6250

\title{
Non-classical selectivities in the reduction of alkenes by cobalt-mediated hydrogen atom transfer $\dagger$
}

\author{
Xiaoshen Ma and Seth B. Herzon* \\ Classical methods for alkene hydrogenation typically reduce less-substituted or more-strained alkenes, or \\ those in proximity to a directing group, most rapidly. Here we describe a cobalt-mediated hydrogenation \\ protocol that provides complementary selectivities in the reduction of several classes of olefins and \\ alkynes. The selectivity of this reduction derives from a hydrogen atom transfer mechanism, which favors \\ the generation of the more stable alkylradical intermediate. We also report the first alkene \\ hydrobromination, hydroiodination, and hydroselenylation by a hydrogen atom transfer process.
}

Received 8th July 2015

Accepted 19th August 2015

DOI: $10.1039 /$ c5sc02476e

www.rsc.org/chemicalscience

coordination (Scheme 1d). ${ }^{52}$ However, for this approach to be successful, the steric encumbrance of the catalyst needs to be minimized to allow the radical-stabilizing effect to dominate. ${ }^{53}$

To facilitate analysis, 2-methylallyl 4-methoxybenzoate (1a) and an equimolar amount of allyl 4-methoxybenzyl ether (1b) were employed as substrates (Table 1). After some experimentation, we found that treatment of a solution of $\mathbf{1 a}$ and $\mathbf{1 b}$ in $n$-propanol with $\mathrm{Co}$ (acac) $)_{2}$ (1.0 equiv.), TBHP (2.0 equiv.), DHB (10 equiv.), and triethylsilane (10 equiv.) formed the products $2 \mathbf{a}$ and $2 \mathbf{b}$ in $71 \%$ and $14 \%$ yields after $135 \mathrm{~min}$ (5.1: 1.0 ratio of 2a : $\mathbf{2 b}$, entry 1). We attempted to improve the selectivity by of metal-catalyzed hydrogen atom transfer reduction had been described, ${ }^{45,46}$ these were the first to proceed with unactivated alkenes as substrates under mild conditions. In the reduction of alkenyl halides, the halogen substituent is thought to control selectivity by biasing the first hydrogen atom transfer toward the generation of a stabilized $\alpha$-haloalkylradical intermediate (Scheme 1b). This mechanism avoids alkylmetal intermediates, which can lead to hydrodehalogenation products. ${ }^{47}$ Shenvi and co-workers subsequently reported a practical method for alkene isomerization and cycloisomerization by hydrogen atom transfer. ${ }^{48}$

The rates of hydrogen atom transfer to alkenes depend upon the stability of the resulting alkylradical intermediate. ${ }^{49,50}$ These data and our alkenyl halide reduction led us to test whether the factors governing hydrogen atom transfer to alkenes could be exploited to obtain non-classical selectivities in alkene hydrogenation (Scheme 1c). Such selectivity would complement traditional approaches, which rely on the higher reactivity of strained and less-hindered alkenes ${ }^{51}$ or functional group

Department of Chemistry, Yale University, New Haven, Connecticut 06520, USA. E-mail: seth.herzon@yale.edu

$\dagger$ Electronic supplementary information (ESI) available: Detailed experimental procedures and characterization data for all new compounds. See DOI: $10.1039 / \mathrm{c} 5 \mathrm{sc} 02476 \mathrm{e}$
Scheme 1 (a) Alkene hydrofunctionalization by hydrogen atom transfer; (b) the hydrogen atom transfer reduction of alkenyl halides to alkyl halides proceeds via selective addition to form a halogen-stabilized alkylradical intermediate; (c) proposed selectivity in hydrogen atom transfer reduction. (d) Classical trends in hydrogenation selectivity.

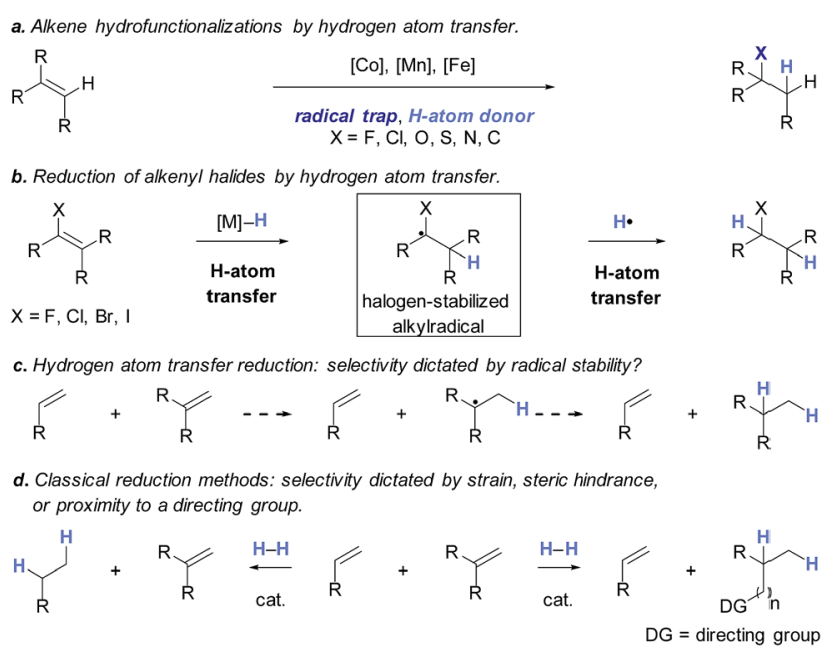


decreasing the reaction temperature, but low conversion was observed (entry 2). Alternatively, when the amount of $\mathrm{Co}(\mathrm{acac})_{2}$ and TBHP were reduced to $50 \mathrm{~mol} \%$, the conversion of $1 \mathrm{a}$ was $72 \%$, but only $31 \%$ of 2 a was obtained, suggesting decomposition of the alkylradical (entry 3). The reactions in entries 1 and 2 were conducted under air in a flask sealed with a septum and pierced with a 16-gauge needle. Conducting the hydrogenation in an open flask enhanced the rate (30 vs. 135-180 min) but diminished selectivity $(63 \%$ and $17 \%$ yield of $2 \mathbf{a}$ and $2 \mathbf{b}$, respectively, entry 4). Interestingly, reducing the amount of $\mathrm{Co}(\mathrm{acac})_{2}$ and TBHP to $25 \mathrm{~mol} \%$ decreased the conversion of $\mathbf{1 b}$ but the major product was the alcohol $3(69 \%$, entry 5$)$. The basis for the difference in product selectivity between entries 4 and 5 is not known, but the production of $\mathbf{3}$ is consistent with earlier reports describing the formal Markovnikov hydration of alkenes by Mukaiyama and co-workers. ${ }^{15,19}$ We posited that higher selectivity and yields could be achieved under an inert atmosphere, as the catalyst would be less activated and the alkylradical intermediate would be less likely to undergo decomposition. When the reaction was conducted under argon, useful selectivities were observed $(4.9: 1.0)$, but the conversion of 1a was incomplete (81\%, entry 6). Warming to $50{ }^{\circ} \mathrm{C}$ increased conversion with only a minor decrease in selectivity ( $4.4: 1.0$ ratio of $2 \mathbf{a}: 2 \mathbf{b}$, entry 7 ). Slow addition of TBHP (syringe pump) provided a 91\% yield of 2a with $4.6: 1.0$ selectivity (entry 8). As the conditions of entry 1 provided the highest selectivity and those of entry 8 afforded the highest yield, both were employed in the investigation of the scope (referred to as conditions A and B, respectively). Other hydrogen atom donors were ineffective. Reduction using manganese tris(dipivaloylmethane) $)^{44}$ was non-selective (see Table S3†).

The experiments in Table 2 establish the relative reactivity of several alkene and alkene-alkyne pairs. For each substrate pair, the condition affording higher selectivities is shown (for complete data, see Table S2 $\dagger$ ). These data show that useful levels of selectivity can be obtained for eight pairs of unsaturated substrates. For example, entries 1 and 2 show that 2,2disubstituted alkenes are reduced selectively over $\alpha$-olefins, and that allylic substituents such as esters, bulky silyl ethers, or alkyl ethers do not significantly influence selectivity. The results in entries 3 and 4 show that bromo- and chloroalkenes are reduced more readily than $\alpha$-olefins, which reflects the additional stabilization afforded by the halogen. ${ }^{54}$ It is noteworthy that reduction of the bromoalkene 1e is complete within $20 \mathrm{~min}$ while $\sim 2 \mathrm{~h}$ are required to achieve conversion of the dialkylsubstituted alkene 1a. The cyclic 2,2-disubstituted alkene 1f was also reduced with comparable selectivity over the $\alpha$-olefin $4 a$ (entry 5). In accord with these data and the mechanistic hypothesis shown in Scheme 1, 2, 2-disubstituted alkenes are reduced more readily than 1,2-disubstituted alkenes (entries 6 and 7). Heterogeneous hydrogenation catalysts typically reduce alkynes faster than alkenes, ${ }^{51}$ but this reduction method provides high levels of selectivity for 2,2-disubstituted alkenes over internal alkynes (entry 8). Trisubstituted alkenes are reduced with modest selectivity over $\alpha$-olefins (entries 11 and 12), but are reduced with higher selectivities over trans- or cis1,2-disubstituted alkenes (entries 13 and 14, respectively). Styrenyl and terminal arylalkynes undergo rapid decomposition to unidentified products (entries 15 and 16) and fluoroalkenes react slowly under these conditions (entry 17). To confirm that these conditions are effective in a polyfunctional setting, we evaluated the reduction of the diene 9 (Scheme 2). These conditions resulted in $72 \%$ reduction of the 2,2-disubstituted alkene and $9 \%$ reduction of the 1,2-disubstituted alkene $(8.0: 1.0$ selectivity).

To benchmark these data, the relative reactivity of six classes of unsaturated substrates were examined under heterogeneous conditions (Table 3 , for additional conditions, see Table S3†). As expected, the less-hindered alkene (or alkyne) was reduced preferentially. Thus, whereas classical hydrogenation

Table 1 Optimization of the reduction mediated by $\mathrm{Co}(\mathrm{acac})_{2}{ }^{a}$

\begin{tabular}{|c|c|c|c|c|c|c|c|}
\hline & $\underset{1 \mathrm{a}}{\mathrm{PMPCO}_{2}}$ & $\begin{array}{c}\mathrm{Co(acac})_{2}(1.0 \text { equiv) } \\
\underset{\operatorname{TBHP}(2.0 \text { equiv) }}{\mathrm{DHB}(10 \text { equiv) }}\end{array} \longrightarrow$ & $\underbrace{\mathrm{PMPCO}_{2} \overbrace{\mathrm{CHBO}}^{\mathrm{CH}_{3}} \mathrm{CH}_{3}}_{\mathbf{2 b}} \mathrm{CH}_{3}$ & 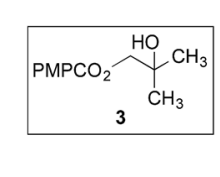 & & & \\
\hline Entry & Variation from above & Time & Conv. 1a & Yield 2a & Conv. 1b & Yield $\mathbf{2 b}$ & $2 \mathbf{a}: 2 \mathbf{b}$ \\
\hline 2 & $0{ }^{\circ} \mathrm{C}$ & $300 \mathrm{~min}$ & $<5 \%$ & $<1 \%$ & $7 \%$ & $<1 \%$ & $-^{b}$ \\
\hline 3 & $\mathrm{Co}(\mathrm{acac})_{2}$, TBHP (50 mol\% each) & $180 \mathrm{~min}$ & $72 \%$ & $31 \%$ & $18 \%$ & $<1 \%$ & $-^{b}$ \\
\hline 4 & Open flask & $30 \mathrm{~min}$ & $>95 \%$ & $63 \%$ & $56 \%$ & $17 \%$ & $3.7: 1.0$ \\
\hline 5 & Co(acac $)_{2}$, TBHP (25 mol\% each), open flask & $180 \mathrm{~min}$ & $75 \%$ & $25 \%(69 \% \text { of } 3)^{c}$ & $6 \%$ & $<1 \%$ & $-^{b}$ \\
\hline 6 & Argon & $360 \mathrm{~min}$ & $81 \%$ & $69 \%$ & $33 \%$ & $14 \%$ & $4.9: 1.0$ \\
\hline 7 & Argon, $50{ }^{\circ} \mathrm{C}$ & $120 \mathrm{~min}$ & $>95 \%$ & $80 \%$ & $25 \%$ & $18 \%$ & $4.4: 1.0$ \\
\hline
\end{tabular}

${ }^{a}$ Reactions employed $250 \mu \mathrm{mol}$ each of $\mathbf{1 a}$ and $\mathbf{1 b}$. Conversions and yields were determined by ${ }^{1} \mathrm{H}$ NMR spectroscopy using mesitylene or 1,3,5-trimethoxybenzene as an internal standard. ${ }^{b}$ The ratio of $2 \mathbf{a}: \mathbf{2} \mathbf{b}$ could not be determined due to the absence of $2 \mathbf{a}$ and/or $2 \mathbf{b}$ in the ${ }^{1} \mathrm{H}$ NMR spectrum of the unpurified product mixture. ${ }^{c} 69 \%$ of 3 was isolated after purification by flash-column chromatography. 
Table 2 Relative reactivity of different alkene or alkene-alkyne pairs toward reduction by Co(acac) 2

\begin{tabular}{|c|c|c|c|c|c|c|}
\hline Entry & Target substrate & Conditions and yield ${ }^{a}$ & Competition substrate & Conversion $^{b}$ & Yield $^{b}$ & $\begin{array}{l}\text { Ratio of reduction } \\
\text { products }\end{array}$ \\
\hline 1 & $1 \mathrm{a}$ & A: $79^{b} \%$ & $1 \mathrm{c}$ & $17 \%$ & $14 \%$ & $5.6: 1.0$ \\
\hline 2 & $1 \mathrm{a}$ & A: $86 \%$ & $4 a$ & $17 \%$ & $12 \%$ & $7.2: 1.0$ \\
\hline 3 & $1 d$ & A: $79 \%$ & $4 a$ & $15 \%$ & $15 \%$ & $5.3: 1.0$ \\
\hline 4 & & A: $71 \%$ & $4 a$ & $5 \%$ & $-^{c}$ & $14: 1.0^{d}$ \\
\hline 5 & If & A: $78 \%$ & $4 a$ & $17 \%$ & $17 \%$ & $4.6: 1.0$ \\
\hline 6 & 1a & B: $96 \%$ & $4 \mathrm{~b}$ & $11 \%$ & $11 \%$ & $8.7: 1.0$ \\
\hline 7 & $1 \mathrm{a}$ & A: $70 \%$ & TBDP & $14 \%$ & $8 \%$ & $8.8: 1.0$ \\
\hline 8 & $1 \mathrm{a}$ & B: $93 \%$ & $\mathrm{CH}_{3}$ & $12 \%$ & $-^{c}$ & $7.8: 1.0^{d}$ \\
\hline 9 & $1 \mathbf{a}$ & B: $89 \%$ & $4 e$ & $30 \%$ & $-^{c}$ & $3.0: 1.0^{d}$ \\
\hline 10 & $1 \mathrm{a}$ & B: $90 \%$ & $\mathrm{CH}_{3}$ & $22^{e} \%$ & $-^{c}$ & $4.1: 1.0^{d}$ \\
\hline 11 & $1 \mathrm{~g}$ & A: $92 \%$ & $4 a$ & $46 \%$ & $46 \%$ & $2.0: 1.0$ \\
\hline 12 & $1 \mathrm{~h}$ & A: $95 \%$ & $4 a$ & $64 \%$ & $64 \%$ & $1.5: 1.0$ \\
\hline 13 & & A: $86 \%$ & $4 \mathrm{~b}$ & $22 \%$ & $18 \%$ & $4.8: 1.0$ \\
\hline 14 & $1 \mathrm{~g}$ & A: $90 \%$ & TBDP & $35 \%$ & $28 \%$ & $3.2: 1.0$ \\
\hline 15 & $1 \mathrm{a}$ & A: $95 \%$ & $4 \mathrm{~g}$ & $93^{e_{0}} \%$ & $-^{f}$ & $1.0: 1.0^{d}$ \\
\hline 16 & $1 \mathrm{a}$ & A: $55 \%$ & & $>95^{e} \%$ & $-^{f}$ & $1.0: 1.7^{d}$ \\
\hline
\end{tabular}


Table 2 (Contd.)

Entry

17<smiles>C=C(F)COC(=O)NNC#N</smiles>

$1 i$
A: $62 \%$

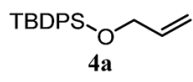

$82 \%$
Ratio of reduction products

\begin{abstract}
${ }^{a}$ Yields refer to purified products isolated by flash-column chromatography, unless otherwise noted. Condition A: Co(acac) 2 (1 equiv.), TBHP (1-8 equiv.), 1,4-DHB (10 equiv.), $\mathrm{Et}_{3} \mathrm{SiH}$ (10 equiv.), $n$ - $\mathrm{PrOH}(0.3 \mathrm{M})$, air, $24^{\circ} \mathrm{C}$. Condition B: $\mathrm{Co}(\mathrm{acac})_{2}(1$ equiv.), TBHP (0.97-1.28 equiv., slow addition), 1,4-DHB (10 equiv.), $\mathrm{Et}_{3} \mathrm{SiH}$ (10 equiv.), $n$-PrOH $(0.3 \mathrm{M})$, argon, $40{ }^{\circ} \mathrm{C}$. The amount of TBHP varies among substrates, see the ESI. ${ }^{b}$ Determined by ${ }^{1} \mathrm{H}$ NMR spectroscopy using mesitylene as an internal standard. ${ }^{c}$ Competition substrate was converted to unidentified products. ${ }^{d}$ Ratios are calculated as the yield of the target substrate versus the conversion of the competition substrate. ${ }^{e}$ Conversion determined by ${ }^{19} \mathrm{~F}$ NMR with hexafluorobenzene as an internal standard. ${ }^{f}$ Decomposition was observed.
\end{abstract}

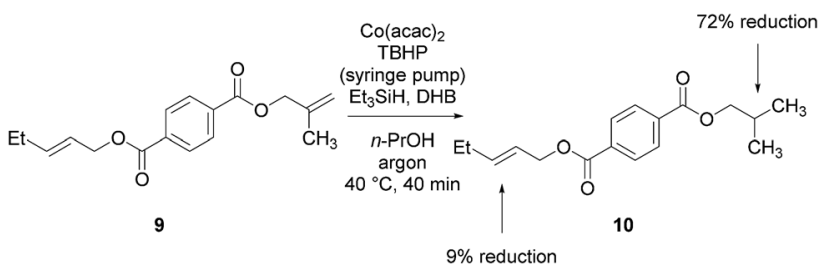

Scheme 2 Hydrogenation of the diene 9.

Table 3 Reduction selectivities under classical and hydrogen atom transfer conditions ${ }^{a}$

\begin{tabular}{|c|c|c|c|}
\hline & $\mathrm{Co}(\mathrm{acac})_{2}$ & $\mathrm{H}_{2} / \mathrm{Pd}-\mathrm{C}$ & \\
\hline & $5.1: 1.0$ & $1.0: 4.4$ & $\mathrm{R} \mathrm{O}$ \\
\hline & $8.7: 1.0$ & $1.0: 1.9$ & $R_{0}$ \\
\hline & $8.8: 1.0$ & $1.0: 1.3$ & $\mathrm{R}_{\mathrm{O}} \mathrm{O}^{\prime}$ \\
\hline $\mathrm{CH}_{\mathrm{CH}_{3}}^{\mathrm{CH}_{3}}$ & $4.8: 1.0$ & $1.0: 8.0$ & $\mathrm{R}_{0} \mathrm{O}$ \\
\hline $\mathrm{O}=\mathrm{CH}_{3}$ & $3.2: 1.0$ & $1.0: 3.3$ & \\
\hline $\mathrm{CH}_{3}$ & $7.8: 1.0$ & $1.0: 2.5$ & \\
\hline
\end{tabular}

${ }^{a}$ For heterogeneous hydrogenation conditions, $\mathrm{R}=\mathrm{PMPCO}_{2}$ (see the ESI).

conditions typically favor reaction of the most accessible (leastsubstituted) alkene, the hydrogen atom transfer reduction we have developed reverses this well-established trend.

Finally, we extended these studies toward the first hydrobromination, hydroiodination, and hydroselenation reactions that proceed by hydrogen atom transfer (Table 4). These experiments find important precedent in the work of Carreira and co-workers, who developed the first hydrochlorination of alkenes by hydrogen atom transfer. ${ }^{32}$ Here we evaluated a range of bromine, iodine, and selenium atom donors under our hydrogen atom transfer conditions (Table S4 $\dagger$ ). We found that addition of $p$-toluenesulfonyl bromide, diiodomethane, or Se-phenyl 4-methylbenzenesulfonoselenoate formed the desired hydrofunctionalization products. The hydrobromination and hydroselenation reactions provided high yields of products for $\alpha-, 2,2-$, and trisubstituted olefins, but the hydroiodination of $\alpha$ - and trisubstituted alkenes did not

Table 4 Hydrobromination, hydroiodination, and hydroselenation of alkenes and alkenyl halides ${ }^{a}$

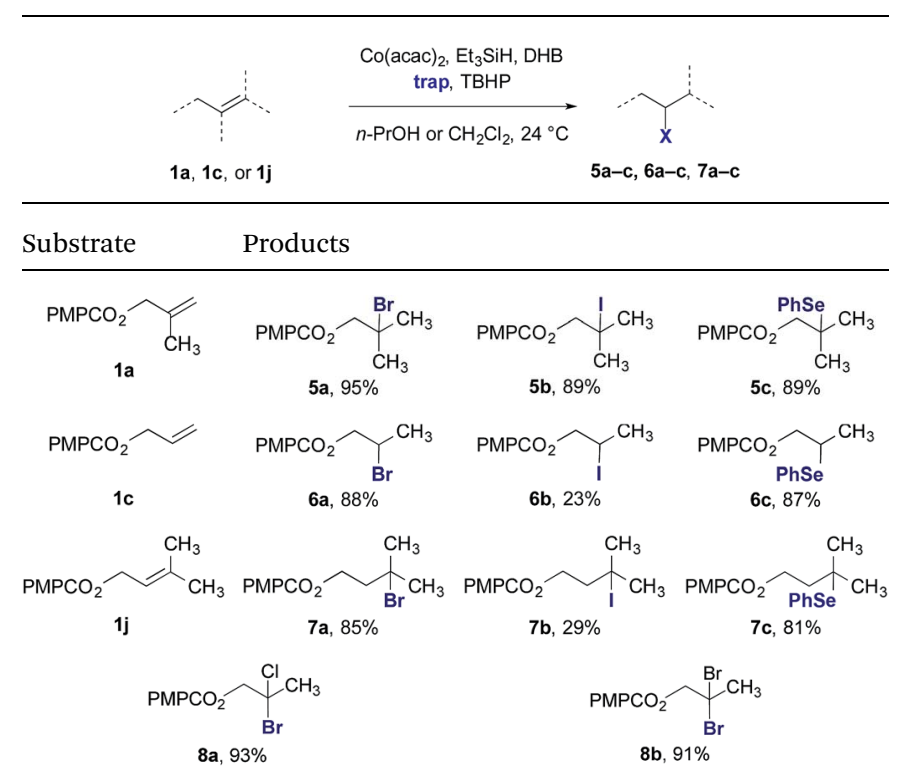

${ }^{a}$ Yields refer to purified products isolated by flash-column chromatography. Hydrobromination: $\mathrm{Co}(\mathrm{acac})_{2}$ (1 equiv.), TBHP (1 equiv.), 1,4-DHB (3.75 equiv. for unfunctionalized alkenes, omitted for alkenyl halides), $\mathrm{Et}_{3} \mathrm{SiH}$ (10 equiv.), tosyl bromide (2.5 equiv.), $n$-PrOH $(0.3 \mathrm{M})$ for unfunctionalized alkenes, DCM $(0.3 \mathrm{M})$ for alkenyl halides, argon, $24{ }^{\circ} \mathrm{C}$. Hydroiodination: $\mathrm{Co}(\mathrm{acac})_{2}$ (1 equiv.), TBHP (1 equiv.), 1,4DHB (3.75 equiv.), $\mathrm{Et}_{3} \mathrm{SiH}$ (10 equiv.), diiodomethane (15 equiv.), DCM $(0.3 \mathrm{M})$, argon, $24{ }^{\circ} \mathrm{C}$. Hydroselenation: $\mathrm{Co}(\mathrm{acac})_{2}$ ( 1 equiv.), TBHP (1 equiv.), 1,4-DHB (3.75 equiv.), $\mathrm{Et}_{3} \mathrm{SiH}$ (10 equiv.), Se-phenyl 4methylbenzenesulfonoselenoate (2.5 equiv.), $n$ - $\mathrm{PrOH}(0.3 \mathrm{M})$, argon, $24{ }^{\circ} \mathrm{C}$. 
proceed to completion. Application of the hydrobromination reaction to alkenyl halides formed the geminal dihalides $\mathbf{8 a}$ and $\mathbf{8 b}$ in high yield.

\section{Conclusions}

In summary, we have shown that hydrogen atom transfer reduction provides selectivities that complement classical methods in the reduction of several alkene and alkene-alkyne pairs. In addition, we have described the first hydrobromination, hydroiodination, and hydroselenation of alkenes that proceed by hydrogen atom transfer. We believe that these methods constitute useful additions to the burgeoning area of practical hydrogen atom transfer reactions.

\section{Acknowledgements}

Financial support from the National Science Foundation (CHE1151563 ) is gratefully acknowledged.

\section{Notes and references}

1 D. C. Eisenberg and J. R. Norton, Isr. J. Chem., 1991, 31, 5566.

2 A. Gansäuer, L. Shi, M. Otte, I. Huth, A. Rosales, I. SanchoSanz, N. Padial and J. E. Oltra, Hydrogen Atom Donors: Recent Developments. Radicals in Synthesis III, Springer, Berlin Heidelberg, 2012.

3 R. L. Sweany and J. Halpern, J. Am. Chem. Soc., 1977, 99, 8335-8337.

4 J. A. Roth and M. Orchin, J. Organomet. Chem., 1979, 182, 299-311.

5 R. L. Sweany, D. S. Comberrel, M. F. Dombourian and N. A. Peters, J. Organomet. Chem., 1981, 216, 37-63.

6 T. E. Nalesnik, J. H. Freudenberger and M. Orchin, J. Mol. Catal., 1982, 16, 43-49.

7 J. A. Roth, P. Wiseman and L. Ruszala, J. Organomet. Chem., 1982, 240, 271-275.

8 F. Ungvary and L. Marko, Organometallics, 1982, 1, 11201125.

9 J. W. Connolly, Organometallics, 1984, 3, 1333-1337.

10 J. F. Garst, T. M. Bockman and R. Batlaw, J. Am. Chem. Soc., 1986, 108, 1689-1691.

11 M. J. Thomas, T. A. Shackleton, S. C. Wright, D. J. Gillis, J. P. Colpa and M. C. Baird, J. Chem. Soc., Chem. Commun., 1986, 312-314.

12 B. Wassink, M. J. Thomas, S. C. Wright, D. J. Gillis and M. C. Baird, J. Am. Chem. Soc., 1987, 109, 1995-2002.

13 J. Choi, M. E. Pulling, D. M. Smith and J. R. Norton, J. Am. Chem. Soc., 2008, 130, 4250-4252.

14 A. Nishinaga, T. Yamada, H. Fujisawa, K. Ishizaki, H. Ihara and T. Matsuura, J. Mol. Catal., 1988, 48, 249-264.

15 T. Mukaiyama, S. Isayama, S. Inoki, K. Kato, T. Yamada and T. Takai, Chem. Lett., 1989, 449-452.

16 S. Inoki, K. Kato, T. Takai, S. Isayama, T. Yamada and T. Mukaiyama, Chem. Lett., 1989, 515-518.

17 S. Isayama and T. Mukaiyama, Chem. Lett., 1989, 569-572.
18 S. Isayama and T. Mukaiyama, Chem. Lett., 1989, 573-576. 19 S. Isayama and T. Mukaiyama, Chem. Lett., 1989, 1071-1074.

20 K. Kato, T. Yamada, T. Takai, S. Inoki and S. Isayama, Bull. Chem. Soc. Jpn., 1990, 63, 179-186.

21 S. Isayama, Bull. Chem. Soc. Jpn., 1990, 63, 1305-1310.

22 K. Kato and T. Mukaiyama, Chem. Lett., 1992, 1137-1140.

23 P. Magnus, A. H. Payne, M. J. Waring, D. A. Scott and V. Lynch, Tetrahedron Lett., 2000, 41, 9725-9730.

24 J. Waser and E. M. Carreira, J. Am. Chem. Soc., 2004, 126, 5676-5677.

25 J. Waser and E. M. Carreira, Angew. Chem., Int. Ed., 2004, 43, 4099-4102.

26 J. Waser, J. C. González-Gómez, H. Nambu, P. Huber and E. M. Carreira, Org. Lett., 2005, 7, 4249-4252.

27 J. Waser, H. Nambu and E. M. Carreira, J. Am. Chem. Soc., 2005, 127, 8294-8295.

28 T. Tokuyasu, S. Kunikawa, K. J. McCullough, A. Masuyama and M. Nojima, J. Org. Chem., 2005, 70, 251-260.

29 J. Waser, B. Gaspar, H. Nambu and E. M. Carreira, J. Am. Chem. Soc., 2006, 128, 11693-11712.

30 B. Gaspar and E. M. Carreira, Angew. Chem., Int. Ed., 2007, 46, 4519-4522.

31 B. Gaspar, J. Waser and E. M. Carreira, Synthesis, 2007, 38393845 .

32 B. Gaspar and E. M. Carreira, Angew. Chem., Int. Ed., 2008, 47, 5758-5760.

33 B. Gaspar and E. M. Carreira, J. Am. Chem. Soc., 2009, 131, 13214-13215.

34 T. Taniguchi, N. Goto, A. Nishibata and H. Ishibashi, Org. Lett., 2010, 12, 112-115.

35 V. Girijavallabhan, C. Alvarez and F. G. Njoroge, J. Org. Chem., 2011, 76, 6442-6446.

36 E. K. Leggans, T. J. Barker, K. K. Duncan and D. L. Boger, Org. Lett., 2012, 14, 1428-1431.

37 T. J. Barker and D. L. Boger, J. Am. Chem. Soc., 2012, 134, 13588-13591.

38 H. Shigehisa, T. Aoki, S. Yamaguchi, N. Shimizu and K. Hiroya, J. Am. Chem. Soc., 2013, 135, 10306-10309.

39 H. Shigehisa, E. Nishi, M. Fujisawa and K. Hiroya, Org. Lett., 2013, 15, 5158-5161.

40 J. C. Lo, Y. Yabe and P. S. Baran, J. Am. Chem. Soc., 2014, 136, 1304-1307.

41 J. C. Lo, J. Gui, Y. Yabe, C.-M. Pan and P. S. Baran, Nature, 2014, 516, 343-348.

42 J. Gui, C.-M. Pan, Y. Jin, T. Qin, J. C. Lo, B. J. Lee, S. H. Spergel, M. E. Mertzman, W. J. Pitts, T. E. la Cruz, M. A. Schmidt, N. Darvatkar, S. R. Natarajan and P. S. Baran, Science, 2015, 348, 886-891.

43 S. M. King, X. Ma and S. B. Herzon, J. Am. Chem. Soc., 2014, 136, 6884-6887.

44 K. Iwasaki, K. K. Wan, A. Oppedisano, S. W. M. Crossley and R. A. Shenvi, J. Am. Chem. Soc., 2014, 136, 1300-1303.

45 S.-K. Chung, J. Org. Chem., 1979, 44, 1014-1016.

46 P. Magnus, M. J. Waring and D. A. Scott, Tetrahedron Lett., 2000, 41, 9731-9733.

47 A. A. Peterson, K. A. Thoreson and K. McNeill, Organometallics, 2009, 28, 5982-5991. 
48 S. W. M. Crossley, F. Barabé and R. A. Shenvi, J. Am. Chem. Soc., 2014, 136, 16788-16791.

49 L. Tang, E. T. Papish, G. P. Abramo, J. R. Norton, M.-H. Baik, R. A. Friesner and A. Rappé, J. Am. Chem. Soc., 2003, 125, 10093-10102.

50 J. Choi, L. Tang and J. R. Norton, J. Am. Chem. Soc., 2007, 129, 234-240.

51 R. L. Augustine, Heterogeneous Catalysis for the Synthetic Chemist, Taylor \& Francis, 1995.
52 A. H. Hoveyda, D. A. Evans and G. C. Fu, Chem. Rev., 1993, 93, 1307-1370.

53 For example, the bulky complex manganese tris(dipivaloylmethane) mediates the reduction of $\alpha$-olefins faster than 2,2-disubstituted or trisubstituted alkenes, despite the lower stability of the secondary alkylradical intermediate, see ref. 44.

54 Y.-R. Luo, Comprehensive Handbook of Chemical Bond Energies, CRC Press, Boca Raton, 2007. 